Research papers

\title{
A semi-analytical generalized Hvorslev formula for estimating riverbed hydraulic conductivity with an open-ended standpipe permeameter
}

\author{
Sergey P. Pozdniakov ${ }^{\mathrm{a}}$, Ping Wang ${ }^{\mathrm{b}, *}$, Mikhail V. Lekhov ${ }^{\mathrm{a}}$ \\ a Department of Hydrogeology, Lomonosov Moscow State University, GSP-1, Leninskie Gory, Moscow 119899, Russia \\ ${ }^{\mathrm{b}}$ Key Laboratory of Water Cycle E Related Land Surface Processes, Institute of Geographic Sciences and Natural Resources Research, Chinese Academy of Sciences, 11A, Datun \\ Road, Chaoyang District, Beijing 100101, PR China
}

\section{A R T I C L E I N F O}

\section{Article history:}

Received 26 January 2016

Received in revised form 4 June 2016

Accepted 27 June 2016

Available online 28 June 2016

This manuscript was handled by $C$.

Corradini, Editor-in-Chief, with the

assistance of Philip Brunner, Associate Editor

\section{Keywords:}

Surface-groundwater exchange

Hydraulic conductivity

Slug tests

Riverbed sediments

Hydraulic resistance

Superposition

\begin{abstract}
A B S T R A C T
The well-known Hvorslev (1951) formula was developed to estimate soil permeability using single-well slug tests and has been widely applied to determine riverbed hydraulic conductivity using in situ standpipe permeameter tests. Here, we further develop a general solution of the Hvorslev (1951) formula that accounts for flow in a bounded medium and assumes that the bottom of the river is a prescribed head boundary. The superposition of real and imaginary disk sources is used to obtain a semi-analytical expression of the total hydraulic resistance of the flow in and out of the pipe. As a result, we obtained a simple semi-analytical expression for the resistance, which represents a generalization of the Hvorslev (1951). The obtained expression is benchmarked against a finite-element numerical model of 2-D flow (in $r$ - $z$ coordinates) in an anisotropic medium. The results exhibit good agreement between the simulated and estimated riverbed hydraulic conductivity values. Furthermore, a set of simulations for layered, stochastically heterogeneous riverbed sediments was conducted and processed using the proposed expression to demonstrate the potential associated with measuring vertical heterogeneity in bottom sediments using a series of standpipe permeameter tests with different lengths of pipe inserted into the riverbed sediments.
\end{abstract}

(c) 2016 Elsevier B.V. All rights reserved.

\section{Introduction}

The exchange processes between surface water and groundwater are significant not only in riverbank water resource management (Chen and Chen, 2003; Winter et al., 1998) but also in water quality considerations (Calver, 2001) associated with biogeochemical interactions between streams and surrounding aquifer systems (Hiscock and Grischek, 2002). Riverbed hydraulic conductivity $(K)$ is one of the key factors controlling the magnitude and spatial distribution of surface-groundwater exchange processes (Genereux et al., 2008; Landon et al., 2001), and it may vary over more than eight orders of magnitude, ranging from below $1.0 \times 10^{-9} \mathrm{~m} / \mathrm{s}$ to above $1.0 \times 10^{-2} \mathrm{~m} / \mathrm{s}$ (Calver, 2001), depending on the riverbed sediment materials and textures (Min et al., 2013; Taylor et al., 2013).

Although numerous approaches (e.g., grain-size distribution analysis, Darcy's law-based in-stream tests, environmental tracer experiments, water balance techniques, integrated surfacegroundwater numerical modeling, etc.) have been widely applied

\footnotetext{
* Corresponding author.

E-mail address: wangping@igsnrr.ac.cn (P. Wang).
}

to investigate the hydraulic properties of riverbed sediments (Cheong et al., 2008; Kalbus et al., 2006; Wang et al., 2015), the accurate estimation of riverbed $K$ values remains a challenge. One of the challenging aspects of estimating riverbed $K$ is associated with its high spatial and temporal variability across measurement scales due to heterogeneity in the riverbed sediments (Chen et al., 2010), scouring and depositional processes during flooding events (Dunkerley, 2008; Hatch et al., 2010), and diurnal and seasonal changes in stream flow temperature (Constantz, 1998). Additionally, the successful application of the aforementioned methods is highly dependent on the assumptions and limitations of the applied methods, the specific equipment, and the design of the measurements (Shanafield and Cook, 2014). Therefore, to estimate riverbed $K$, multiple methods are recommended to reduce the method uncertainties involved in many field studies (Fleckenstein et al., 2010).

Field methods, including slug tests, in situ permeameter tests, and seepage-meter measurements, have been widely applied to determine the hydraulic properties of riverbeds. For studying riverbed vertical heterogeneity, a light-oil piezomanometer, which allows to measure very small head differences between surface water and underlying groundwater, was developed by Kennedy 
et al. (2007). Recently, a new type of permeameter was designed to measure two parameters, i.e., vertical flux and hydraulic gradient, simultaneously on site (Lee et al., 2015). These methods are relatively quick, inexpensive and allowing for numerous measurements to be made at many locations (Landon et al., 2001). The falling head slug tests, in which a standpipe (well or piezometer) is filled with river water and the raised water level in the standpipe is immediately allowed to fall while assuming that the general river water level remains constant (Baxter et al., 2003; Hvorslev, 1951), are considered to be a more practical in-stream approach than a permeameter for determining the riverbed $K$ because of their ability to measure much deeper sediments (Landon et al., 2001). Another important advantage of falling head slug tests is that this type of test can evaluate the anisotropy of riverbed sediments using the L-shaped standpipe method (Chen, 2000), which provides in situ measurements of riverbed $K$ in different directions.

Hvorslev (1951) conducted detailed interpretations of field standpipe permeameter tests using different types of piezometers and provided corresponding formulas to calculate the hydraulic conductivity. Hvorslev's falling-head analysis generated accurate vertical hydraulic conductivities of the riverbed in homogenous sediments and layered deposits of low- $K$ sand over high- $K$ sand (Burnette et al., 2016). The analytical solution produced by Hvorslev (1951) highly depends on the shape factor of the installed piezometer $(F)$, which is considered a function of the geometric constants, i.e., the length-to-diameter ratio, of the piezometer (Silvestri et al., 2012). As indicated by Klammler et al. (2011), most existing approaches used to determine $F$ are based only on geometric or mathematical simplifications that neglect the effects of the boundaries of the flow domain. Therefore, the objectives of this study are to: (1) develop a semi-analytical expression for hydraulic resistance of an open-ended standpipe permeameter in the vicinity of a constant head boundary; (2) validate the obtained expression using numerical simulations of the falling head tests in the standpipe permeameter; (3) examine the influence of the natural vertical flow gradient in bottom sediments and medium elastic storage on the falling head test results; and (4) analyse the possibility of determining the hydraulic conductivity profiles of layered bottom sediments using falling head tests in a standpipe permeameter.

\section{Development of an analytical model}

\subsection{A semi-analytical solution for hydraulic resistance}

As shown in Fig. 1, an open-ended cylindrical pipe has a diameter $d$ and a penetration length into the riverbed sediments $L$. Let us assume that the initial water level in the pipe $H_{\text {pipe }}(0)$ is equal to the river water level $H_{\text {riv }}$, i.e., $H_{\text {pipe }}(0)=H_{\text {riv }}$. The water level in the pipe is instantaneously raised to $S_{0}$ above the river water level $H_{r i v}$, and the subsequent raised water level in the pipe relative to the initial water level in the pipe $H_{\text {pipe }}(0)$ is $S(t)$.

A semi-infinite medium with an origin in cylindrical coordinates $(r, z)$ is placed at the upper boundary of the sediments in the centre of the pipe with diameter $d$. The riverbed sediments are assumed to be horizontally anisotropic media, i.e., the saturated hydraulic conductivity at each field point can be characterized by the radial horizontal $\left(K_{r}\right)$ and vertical $\left(K_{z}\right)$ components of hydraulic conductivity tensor $\mathbf{K}$. The coefficient of anisotropy of conductivity can be defined as follows: $\alpha^{2}=K_{z} / K_{r}$.

The equation of unsteady state flow in an anisotropic medium from the pipe into the riverbed sediments, in cylindrical coordinates $(r, z)$, is written as follows, using superposition principles in terms of changes in hydraulic head (Neuman, 1975):

$S_{s} \frac{\partial s}{\partial t}=\frac{1}{r} \frac{\partial}{\partial r} r K_{r} \frac{\partial s}{\partial r}+K_{z} \frac{\partial^{2} s}{\partial z^{2}}$

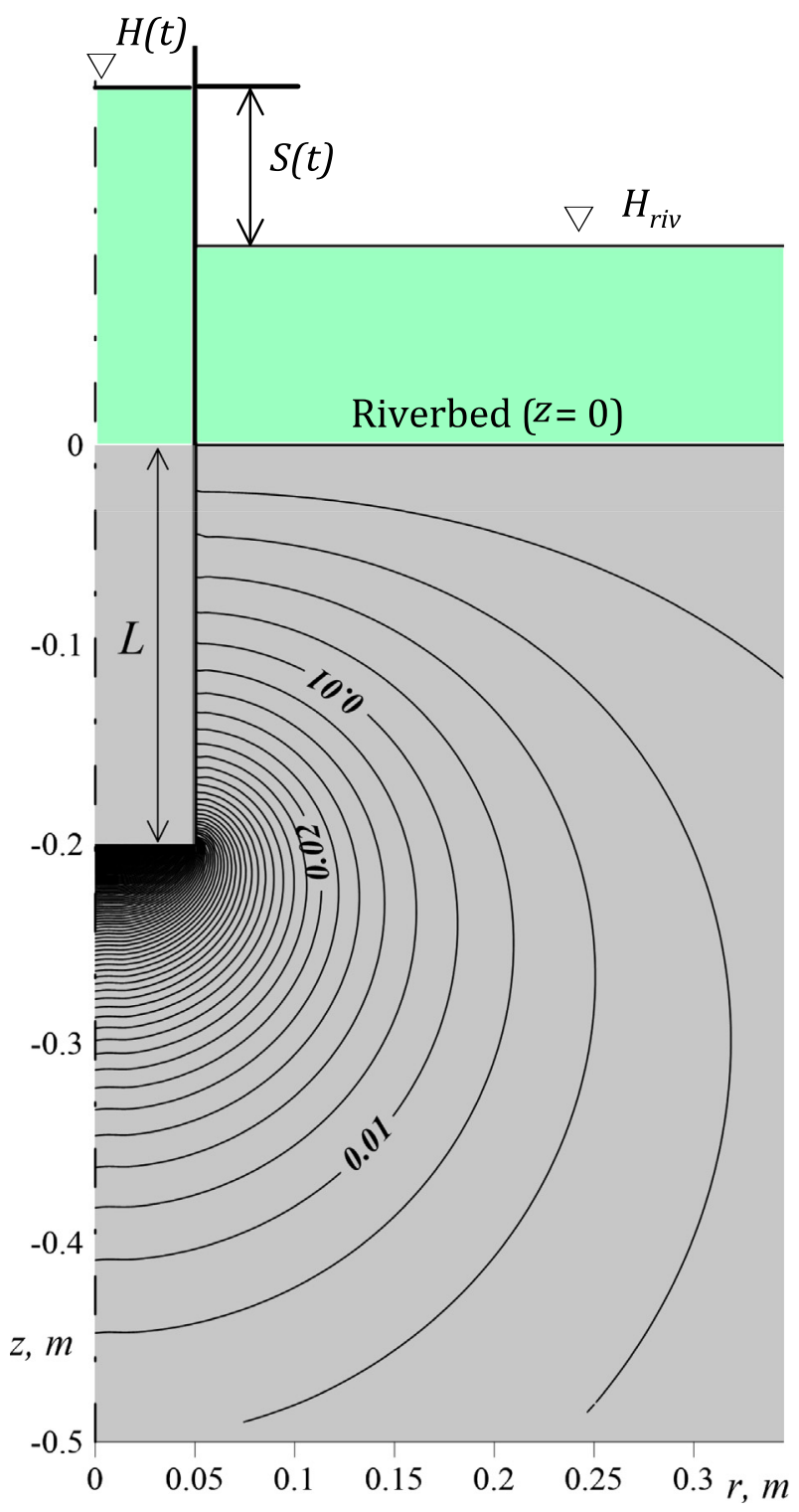

Fig. 1. Cylindrical pipe installed in riverbed sediments with equipotential of groundwater head change in the sediments. $H_{\text {riv }}$ is the river level, $H(t)$ is the water level in the pipe, and $S(t)$ is raised over the $H_{\text {riv }}$ water level in the pipe.

where $S_{s}$ is specific storage, and $s(r, z, t)=H(r, z, t)-H(r, z, 0)$ is the change in hydraulic head $H(r, z, t)$, i.e., its increase above the initial head $H(r, z, 0)$.

The initial condition of Eq. ( 1 ) is $s(r, z)=0$ for $r>d / 2$, and $s=S_{0}$ for $z=0$ and $r<d / 2$ at $t=0 . S_{0}$ is the instantaneous change in the water level inside the pipe above the river water level at $t=0$. The boundary conditions at the wall of the pipe are no-flow conditions. At the top of the sediments inside the pipe, i.e., $z=0$ and $r<d / 2$, the hydraulic head change in the sediments is $s(r, 0, t)=S$ $(t)$. The flow rate from the pipe $Q$ is system-dependent, and it can be found by equating the flow in the pipe to the flow in the medium:

$Q=-\frac{\pi d^{2}}{4} \frac{d S}{d t}=\left.2 \pi \int_{0}^{d / 2} r K_{z} \frac{\partial S}{\partial z}\right|_{z=0} d r$

At the surface $z=0$ outside the pipe $(r>d / 2)$, two types of boundary conditions can generally be considered: a) a no-flow boundary and b) a constant-head $(s=0)$ boundary. Note that the no-flow boundary at the top of the bottom sediments is not 
realistic in real hydrological systems; however, we consider both types of boundary conditions to analyse the scales of different boundary effect on analytical solution.

The goal of this section is to develop a functional relationship between the change in the water level in the pipe $S(t)$ above the initial level $H_{\text {pipe }}(0)$ and the outflow rate from the pipe $Q$ as a function of the hydraulic conductivity of the medium and the following pipe parameters: penetration length $L$ and diameter $d$.

To determine this relationship, we consider the case of incompressible fluid flow in an incompressible medium, i.e., $S_{S}=0$. Next, we apply a method of local hydraulic resistance, which separates the flow into the inner part, i.e., the flow within the pipe filled with sediment, and outer part, i.e., the flow outside the pipe. According to this method, the outflow rate from the pipe $Q$ is associated with the change in water level $S$ and the total hydraulic resistance, which comprises internal resistance and external resistance:

$Q=\frac{S}{R_{\text {in }}(L)+R_{\text {out }}(L)}$,

where $R_{i n}(L)$ is the internal hydraulic resistance of the flow within the pipe and $R_{\text {out }}(L)$ is the external hydraulic resistance of the flow in the medium surrounding the pipe. Let us suppose that the flow lines within the pipe are parallel to the wall of the pipe. Thus, the flow within the pipe can be considered one dimensional in the $z$ direction. In this case, the internal hydraulic resistance can be expressed as the resistance of a one-dimensional flow with length $L$ across an area of $\pi d^{2} / 4$ as follows:

$R_{\text {in }}(L)=\frac{4 L}{K_{z} \pi d^{2}}$,

The external hydraulic resistance is the resistance of divergent flow and depends on the hydraulic conductivity and geometric constants, i.e., the pipe diameter $d$ and the distance from the pipe opening to the boundary $L$. It also depends on the type of boundary condition at $z=0$ (a no-flow boundary or constant-head boundary).

For further development, the coordinates in Eq. (1) can be transformed to obtain the flow equation in an isotropic media with scaled geometry: $r^{\prime}=r \alpha, z^{\prime}=z$, and isotropic hydraulic conductivity $K=\sqrt{K_{r} K_{z}}$. Next, to determine the external resistance, flow in an infinite medium, i.e., $L \rightarrow \infty$, is considered. An exact analytical solution for steady-state flow into an open-ended well with a short screen partially penetrating an isotropic medium was developed by Silvestri et al. (2012). According to Silvestri et al. (2012), the relationship between $S$ and $Q$ for steady state flow in an isotropic unbounded aquifer from an open-ended pipe without a well screen can be expressed in the following form:

$S=\frac{Q}{2.8 K d}$

Taking into account the anisotropy transformation, the external resistance based on Eq. (5) can be written as follows when $L \rightarrow \infty$ :

$R_{\text {out }}(\infty)=\frac{1}{a(\infty) K \alpha d}$,

where $a(\infty)$ is a dimensionless coefficient and is equal to 2.8 .

For $L=0$ and an impermeable upper boundary at $z=0$ and $r>d / 2$, the external resistance can be written in the same form as Eq. (6), but with the dimensionless coefficient $a(0)=2$ using the steady state solution of the thermal resistance of a disk source (Carslaw and Jaeger (Eds.), 1959):

$R_{\text {out }}(0)=\frac{1}{2 K \alpha d}$
For $L=0$ and a constant head boundary at $z=0$ outside the pipe, no analytical results exist for the case of a constant head boundary inside the pipe due to a singularity at the pipe wall as a result of its zero thickness. Carslaw and Jaeger (Eds.) (1959) showed that the total resistances of a constant head and a constant specific flux at the disk surface are very similar. Thus, we applied the wellknown solution by Carslaw and Jaeger (Eds.) (1959) to estimate the thermal resistance of a disk source with a constant specific heat flux rate of $v$. In the present study, the effective disk radius $R=\alpha d / 2$, and the increase in the water level is a function of the disk source radius, which can be expressed as follows:

$S\left(r^{\prime}\right)=\frac{2 v}{\pi K} \int_{0}^{\infty} J_{0}\left(\lambda r^{\prime}\right)(\sin \lambda R-\lambda R \cos \lambda R) \frac{d \lambda}{\lambda^{2}}$,

where $J_{0}(x)$ is a Bessel function of first kind. Then, the average increase in water level over the disk $S_{a v}$ can be defined as $S_{a v}=\frac{1}{\pi R^{2}} \int_{0}^{R} 2 \pi r^{\prime} S\left(r^{\prime}\right) d r^{\prime}=\frac{2}{R^{2}} \int_{0}^{R} r^{\prime} S\left(r^{\prime}\right) d r^{\prime}$. The total flow across the disk is $Q=\pi R^{2} v$, and the resistance can be written in the following form:

$$
\begin{aligned}
R_{\text {out }}(0) & =\frac{S_{a v}}{Q} \\
& =\frac{4}{R^{4} \pi^{2} K} \int_{0}^{R} r^{\prime} \int_{0}^{\infty} J_{0}\left(\lambda r^{\prime}\right)(\sin \lambda R-\lambda R \cos \lambda R) \frac{d \lambda}{\lambda^{2}} d r^{\prime},
\end{aligned}
$$

To evaluate the integrals in Eq. (9), let us define $u=\lambda \cdot R$ and $\bar{r}=r^{\prime} / R=2 r / d$, leading to the following equation:

$R_{\text {out }}(0)=\frac{S_{a v}}{Q}=\frac{4 R^{3}}{R^{4} \pi^{2} K} \int_{0}^{1} \bar{r} \int_{0}^{\infty} J_{0}(u \bar{r})(\sin u-u \cos u) \frac{d u}{u^{2}} d \bar{r}$

Reversing the integration order and simplifying the integration yields:

$$
\begin{gathered}
\int_{0}^{\infty}(\sin u-u \cos u) \frac{1}{u^{2}} \int_{0}^{1} \bar{r} J_{0}(u \bar{r}) d \bar{r} d u \\
=\int_{0}^{\infty} \frac{J_{1}(u)}{u} \frac{\sin u-u \cos u}{u^{2}} d u=\frac{1}{3}
\end{gathered}
$$

Next, substituting $R$ for $d$ yields:

$R_{\text {out }}(0)=\frac{8}{3 d \pi^{2} \alpha K}$

Eq. (12) is analogous to Eq. (7), and in this case $a(0)=\frac{3 \pi^{2}}{8} \approx 3.701$.

Therefore, the external resistance depends on the distance to the boundary and the type of boundary condition at this boundary. The maximum effect of the boundary on the external resistance occurs is for zero total penetration length $L$. If the boundary is non-permeable, then the external resistance increases by a factor of 1.4 relative to that of the infinite medium. If the boundary has a constant head, the external resistance decreases by a factor of 1.325 .

For field tests in riverbed sediments, the boundary is generally set as a constant head boundary. Therefore, the general solution of the external resistance must be obtained for an arbitrary $L$ for a constant head boundary. However, an analytical expression for this general case does not exist. To obtain an approximate expression of the resistance estimation, we apply a well-known method used to estimate the potential of a point source located near a boundary (Bear, 1979). This method assumes the superposition of potentials of real sources and imaginary sinks in an infinite medium and is strictly applicable only for point sources with given flow rates and does not strictly apply to a disk of finite diameter with a given potential. In the far field, the potentials of a disk of finite size and point sources with the same flow rates are similar according to Carslaw and Jaeger (Eds.) (1959), and they tend towards zero with 
increasing distance. Therefore, we assume that the open-ended well is located a sufficient distance away from the boundary. In this case, we can apply the principle of superposition, which is used for point sources, as a weak approximation of the real field $s(r, z)$ of an open-ended well. We use the superposition of two disk sources: one is the real open-ended well that produces the $s_{r}\left(r^{\prime}, z\right)$ field, and the other is an imaginary open-ended well that produces the $s_{i m}\left(r^{\prime}, z\right)$ field. The latter is located at a distance of $2 L$ from the real well and has the opposite sign, i.e., $s\left(r^{\prime}, z\right)=s_{r}\left(r^{\prime}, L-z\right)-s_{i m}\left(r^{\prime}, L+z\right)$. To calculate the hydraulic resistance with superposition, we adopt the well-known solution for a disk source of diameter $d$ with a given potential in an infinite medium (Carslaw and Jaeger (Eds.), 1959), which can be written:

$$
\begin{aligned}
s\left(r^{\prime}, z\right) / S_{d}= & F\left(r^{\prime}, z, d, \alpha\right) \\
= & \frac{2}{\pi} \\
& \times \sin ^{-1}\left(\frac{d \alpha}{\sqrt{\alpha^{2}\left(r^{\prime}-d / 2\right)^{2}+z^{2}}+\sqrt{\alpha^{2}\left(r^{\prime}+d / 2\right)^{2}+z^{2}}}\right)
\end{aligned}
$$

where $S_{d}$ is the potential on the disk source surface, i.e., $S_{d}=Q R_{\text {out }}(\infty)$

Next, we assume that the influence of the imaginary openended well on the real well head at its open end is equal to the mean $s_{i m}\left(r^{\prime}, 2 L\right)$ for $0<r<d / 2$. Averaging Eq. (13) over the disk of the real well at distance $2 L$ from the imaginary disk, we obtain:

$$
\begin{aligned}
f(2 L / \alpha d) & =\frac{2}{d^{2}} \int_{0}^{d / 2} r^{\prime} F\left(r^{\prime}, 2 L, d, \alpha\right) d r^{\prime}=2 \int_{0}^{1} \bar{r} F(\bar{r}, 2 l) d \bar{r} ; l \\
& =\frac{2 L}{\alpha d}
\end{aligned}
$$

Numerically averaging Eq. (14), we find that $f$ is equal to the value obtained from Eq. (13) for $r^{\prime}=0.75 * d / 2$ :

$f(l) \approx \frac{2}{\pi} \sin ^{-1}\left(\frac{2}{\sqrt{0.0625+4 l^{2}}+\sqrt{3.0625+4 l^{2}}}\right)$

Thus, for the far field, i.e., for the case of an open-ended well located a sufficient distance away from the boundary, we can use superposition to write:

$Q=\frac{S_{d}}{R_{\text {out }}(L)} ; R_{\text {out }}(L)=R_{\text {out }}(\infty) /(1-f(l)) ; L \gg d$

Eq. (16) demonstrates that the change in $f(l)$ from 0 to 1 is a transfer function that describes the effect of the boundary on the hydraulic resistance $R_{\text {out }}(L)$ of a point source. For a real open-ended well, let us suppose that the hydraulic resistance is an unknown function of the same transfer function $f(l)$, i.e., $R_{\text {out }}(L)=F[f(l)]$, with known bounds: $F[0]=R_{\text {out }}(\infty)$ and $F[1]=R_{\text {out }}[0]$. By assuming that $F[f(l)]$ is a differentiable function that can be expanded in a Taylor series and by writing a formal expansion of $F[f(l)]$ in a series and retaining only the first two terms of this expansion, i.e., $F[f(l)] \approx F[0]+\frac{\partial F}{\partial f}[f(l)-f(0)]+\ldots$, we obtain a semi-analytical form of the external resistance equation:

$R_{\text {out }}(L) \approx R_{\text {out }}(\infty)+\left[R_{\text {out }}(0)-R_{\text {out }}(\infty)\right] f(l)+\ldots=\frac{1}{a(L) d \alpha K}$

or

$$
a(L)=\frac{a(\infty)}{1-f(l) \cdot\left(1-\frac{a(\infty)}{a(0)}\right)}=\frac{2.8}{1-0.243 f(l)}
$$

Eq. (18) satisfies the explicit well-known boundary values for the external resistance. This agreement is necessary; however, it is not sufficient to assume that the selected transfer function is correct because it cannot guarantee that expansion can be used to calculate the correct value of the external resistance $R_{\text {out }}(L)$ within an acceptable error for any arbitrary proposed value of $L$. This approach is numerically validated in Section 3.

Fig. 2 shows the dependence of the parameter $a$ on the dimensionless distance between a horizontal boundary with a prescribed hydraulic head and an open-ended pipe in an isotropic medium obtained from Eqs. (15) and (18). This dependence reflects the influence of the boundary with a given hydraulic head on the external hydraulic resistance. Generally, we assume that the vertical hydraulic conductivity is less than the horizontal hydraulic conductivity; thus, $\alpha=1$ reflects the maximum influence of the horizontal boundary with a specified hydraulic head on the external resistance for a given penetration depth. As shown in Fig. 2, the impact of a constant head boundary on the hydraulic resistance is less than $5 \%$ for $L>2 d$.

Considering Eqs. (4) and (17), Eq. (3) can be re-written as:

$$
Q=F K_{z} d S
$$

$F=\frac{\pi d}{4 L}\left(1+\frac{\pi d \alpha}{4 L a(L)}\right)^{-1}$

Furthermore, by applying a quasi-stationary model, i.e., assuming that at any time $Q=-\frac{\pi d^{2}}{4} \frac{d S}{d t}=S F K_{z} d$ and for initial time $S=S_{0}$, the expression for the water level dynamics in the pipe can be found to be:

$\ln \frac{S_{0}}{S}=\frac{4 K_{z} F}{\pi d}$

Plotting $\ln \left(S_{0} / S\right)$ versus $t$, reveals a slope of:

$B=\frac{4 K_{z} F}{\pi d}$

By having the parameters of pipe $L$ and $d$, as well as an estimate regarding the coefficient of anisotropy in the porous medium, it is possible to determine the vertical hydraulic conductivity at any installed depth of pipe:

$K_{z}=\frac{B \pi d}{4 F}=B L\left(1+\frac{\pi d \alpha}{4 L a(L)}\right)$,

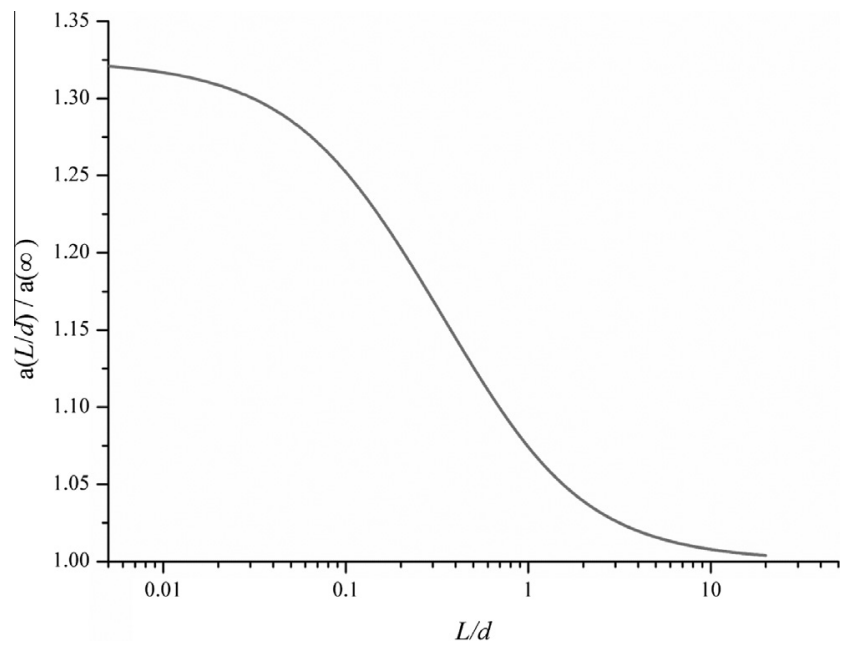

Fig. 2. Dependence of parameter $a$ on the dimensionless distance between a horizontal boundary with a prescribed hydraulic head and an open-ended well. 


\subsection{Effects of a natural vertical flow gradient in bottom sediments and} medium elastic storage

Eq. (22) assumes that the initial value of $S_{0}$ measured in the pipe at an elevation above $H_{\text {riv }}$ is equal to the value measured above the initial water level in the pipe inserted at arbitrary depth $L$, i.e., the ambient pre-test vertical head gradient in the riverbed is zero. If an upward or downward ambient head gradient exists, the ambient hydraulic head at the pipe penetration depth differs from $H_{r i v}$. Thus, the stable water level inside the pipe $H_{\text {pipe }}$ is not $H_{r i v}$; instead, it is either greater or less than $H_{r i v}$, depending on the penetration depth $L$. Therefore, the value of $S_{0}$ measured from the river water level is not the true change in hydraulic head at depth $L$ above the initial value $H_{\text {pipe }}$. A possible practical solution to this problem is waiting for the water level to stabilize within the pipe before the test. For the low riverbed permeability and relatively large diameter pipe, this stabilization may take a long time. To avoid this issue, the extended version of Eq. (20) can be used, taking into account the difference between the head of the stream $H_{\text {riv }}$ and the natural head $H(L)$ at the depth of pipe penetration $L$, i.e., $\Delta H(L)=H_{r i v}-H(L)$ :

$\ln \left(\frac{S_{0}+\Delta H(L)}{S+\Delta H(L)}\right)=\frac{4 K_{z} F}{\pi d} t=B t$

Eq. (23) includes two unknown parameters, $B$ and $\Delta H(L)$, that can generally be found via non-linear regression if the observed curve of $\ln \left(S_{0} / S\right)$ versus time has a non-linear tail. The errors associated with neglecting $\Delta H(L)$ and fitting slope $B^{\prime}$ of Eq. (20) instead of the true slope $B$ of Eq. (23) can be found from the ratio of $\ln (S)$ to $\ln (S+\Delta H(L))$ for small time values:

$B^{\prime} / B \approx 1+\Delta H(L) / S_{0}$

The influence of the external hydraulic resistance on the estimation of vertical hydraulic conductivity is negligible if the second term on the right hand side of Eq. (24) is significantly less than one. For an isotropic aquifer, this term is less than 0.1. Additionally, when $L / d$ is greater than three, the effect on the calculated external resistance is not essential because the main hydraulic resistance is concentrated inside the pipe.

Eq. (22) differs from the associated Hvorslev formula that corresponds to Case E (see Fig. 18, Page 44 in Hvorslev (1951)), which, in the notation of this study, has the following form:

$$
\begin{aligned}
K_{z} & =\ln \frac{S_{1}}{S_{2}}\left(L+\frac{\pi d}{11 \sqrt{\frac{K_{r}}{K_{z}}}}\right) /\left(t_{2}-t_{1}\right)=B L\left(1+\frac{\pi d \alpha}{11 L}\right) ; B \\
& =\ln \frac{S_{1}}{S_{2}} /\left(t_{2}-t_{1}\right),
\end{aligned}
$$

where $S_{1}$ and $S_{2}$ are the head above $H_{\text {riv }}$ at the testing time $t_{1}$ and $t_{2}$.

The denominator produces a value of 11 in Hvorslev (1951) (Eq. (25)), i.e., approximately $4 * 2.8$. However, in our formula (Eq. (22)), this coefficient depends on the distance from the constant head boundary to the open end of the pipe.

The previous solutions assume that the water and the riverbed sediments are incompressible. To estimate the influence of the compressibility of water and sediments, i.e., $S_{s}>0$, we adopt the results of Warrick et al. (1992), who solved the diffusion problem for a disk source in an infinite medium and developed an approximation of the time dependence of the diffusive flux from a disk source. In this paper, the expression developed by Warrick et al. (1992) can be rewritten as:

$Q(t) \approx Q_{\infty}\left(1+\frac{2}{\pi^{3 / 2} \sqrt{T}}\right) ; \quad T=\frac{4 K_{z} t}{S_{s} \alpha d^{2}}$,

where $Q_{\infty}$ is the flow rate for the steady state case (i.e., $S_{s}=0$ ).
Eq. (26) demonstrates that for a characteristic dimensionless time of transient flow $T>10$, the flow rate is close to the steady state rate. Introducing the characteristic dimensionless time based on a falling head test $T_{f}$, which according to Eqs. (20) and (21) is equal to $B t$, the ratio of these times can be written as:

$\frac{T}{T_{f}}=\frac{4 L+\pi d \alpha / a(L)}{d^{2} a S_{s}}$

According to Eq. (27), for a given test time $t$, the dimensionless transient flow time for typical parameters $(L \sim 1 \mathrm{~m}, d=0.1 \mathrm{~m}$, and $S_{s} \sim 10^{-3}-10^{-4} \mathrm{~m}^{-1}$ ) is four orders of magnitude larger than the dimensionless time of the falling test. Therefore, unsteady flow due to elastic storage can be neglected in falling head tests for broad ranges of riverbed hydraulic parameters and pipe dimensions.

\section{Numerical simulation and experimental verification}

\subsection{Model description}

To validate the obtained semi-analytical expression (22), we developed the 1Well code (Lekhov, 2015), which simulates flow surrounding a pumping or injection well with different types of boundary conditions at the well surface, including falling head tests in open-ended pipes. This programme numerically solves Eq. (1) using the finite-element method in $r$ - $z$ coordinates. To take into account the internal resistance of the sediments inside the pipe, this programme uses Robin boundary conditions, which are a weighted combination of Dirichlet and Neumann boundary conditions, for the head and velocity and an integrated water balance at the pipe outlet to find the relationship between the falling head and total outflow from the pipe:

$$
\begin{gathered}
\left.v_{z}(r, t)\right|_{z=L, r<d / 2}=-K_{z} \frac{\partial s_{b}(r, t)}{\partial z}=\beta\left[S(t)-s_{b}(r, t)\right] \\
Q=-\frac{\pi d^{2}}{4} \frac{\partial S(t)}{\partial t}=\left.2 \pi \int_{0}^{d / 2} r v_{z}(r, t)\right|_{z=L} d r
\end{gathered}
$$

where $v_{z}(r, t)$ and $s_{b}(r, t)$ are the velocity and the head change, respectively, in the open-ended pipe that penetrates the sediments to a depth of $L$, and where $\beta=K_{z} / L$ for a pipe filled with sediments and $\beta \rightarrow \infty$ for a pipe without sediments throughout its entire depth L.

We compared the numerical and analytical results for flow in an isotropic medium with hydraulic conductivity $K$ without internal resistance for two cases: (1) $L=0$ and a no-flow boundary for $r>d / 2$ and (2) an infinite medium $(L \rightarrow \infty)$. As shown above, the rigorous relationship between $Q$ and $S$ is as follows for these two cases:

$Q=a(L) K \alpha d S ; \quad a(0)=2 ; \quad a(\infty)=2.8$

Solutions based on the programme 1Well showed that Eq. (29) is performed numerically with an accuracy of $2 \%$, taking into account the spheroid-like head change (Fig. 1) in the mesh design. Along the $r$-axis from $r=0$ to $d / 2$, this mesh for has nine elements of equal area, and element sizes logarithmically increase when $r>d / 2$, with a magnification factor of 1.05 . A logarithmic mesh with a magnification factor of 1.05 is also used for spacing along the $z$-axis. The vertical size of the smallest element is equal to $\mathrm{d} / 2 * 0.05$, and this element is placed at $z=L$. This same mesh design is used for further simulations in support of Eq. (22) discussed below.

\subsection{Numerical assessments}

We performed a set of numerical calculations of falling head tests using 1Well to assess Eq. (22). In all the tests, we used the 
specific storage value simulated using Eq. (1): $S_{S}=5 * 10^{-4} \mathrm{~m}^{-1}$. For each simulation result we produced a plot of $\ln \left(S_{0} / S\right)$ versus $t$ and linear regression to estimate the slope of $\ln \left(S_{0} / S\right)$, i.e., the parameter $B$ (Eq. (21)) and the $K_{z}$ value from Eq. (22). Next, using the estimated $K_{z}$ value, we calculated the dimensionless falling head test time: $T_{f}=K_{z} t /(L+\pi d \alpha / 4 a(L))$.

Fig. 3 shows the estimated $K_{z}$ for an isotropic medium in the form of $\ln \left(S_{0} / S\right)$ versus dimensionless time $T_{f}$. According to Eqs. (20)-(22), if the estimated $K_{z}$ is close to the simulated values, the data from simulations based on different hydraulic parameters should fall near the $Y=X$ line on a plot of $\ln \left(S_{0} / S\right)$ versus dimensionless time $\left(T_{f}\right)$. Fig. 3 shows a very good fit between the experimental data and the $Y=X$ line, suggesting that Eq. (22) is correct for various penetration depths $L$ in an isotropic medium. Fig. 4 shows the same calculation performed for an anisotropic medium. One can see from this figure that the estimation results for an anisotropic medium are also in good agreement with the numerical simulation results for the cases of $\alpha>1$ and $\alpha<1$. No visible effects of the elastic properties of the simulated medium, i.e., using nonzero values of the specific storage based on the $\ln \left(S_{0} / S\right)$ versus $t$ curves, are observed.

Thus, the numerical results obtained for isotropic and anisotropic media over a wide range of $L$ values show that our semianalytical equation (18) for $a(L)$ and transfer function equation (16) can be effectively used for practical purposes in the interpreting field data with Eqs. (21) and (22).

\subsection{Performance considerations in a layered medium}

To study the sensitivity of the falling test results to vertical heterogeneity, we assume a sediment profile with a depth of $2 \mathrm{~m}$ and stochastic changes in $\log K$ that have a vertical exponential correlation $r(z)=\exp \left(-z / \lambda_{z}\right)$, a characteristic length of $\lambda_{z}=0.3 \mathrm{~m}$ and an infinite characteristic correlation length along the $r$-axis. We created three versions of this profile with different values of $\operatorname{Var}(\log K)$, i.e., $0.25,0.5$ and 1 , and a constant vertical resolution of $0.01 \mathrm{~m}$. Then, we simulated falling head tests for each version with a pipe diameter of $d=0.1 \mathrm{~m}$ and different penetration lengths $L$ varying from 0.1 to $1.9 \mathrm{~m}$, with a constant step of $0.1 \mathrm{~m}$. For each profile and each depth, we simulated two types of tests. The first type is the test described above, i.e., the total hydraulic resistance consisting of the internal and external resistances. The second type is a simulation with zero internal resistance, i.e., we assume that the sediments are removed from the pipe before testing.

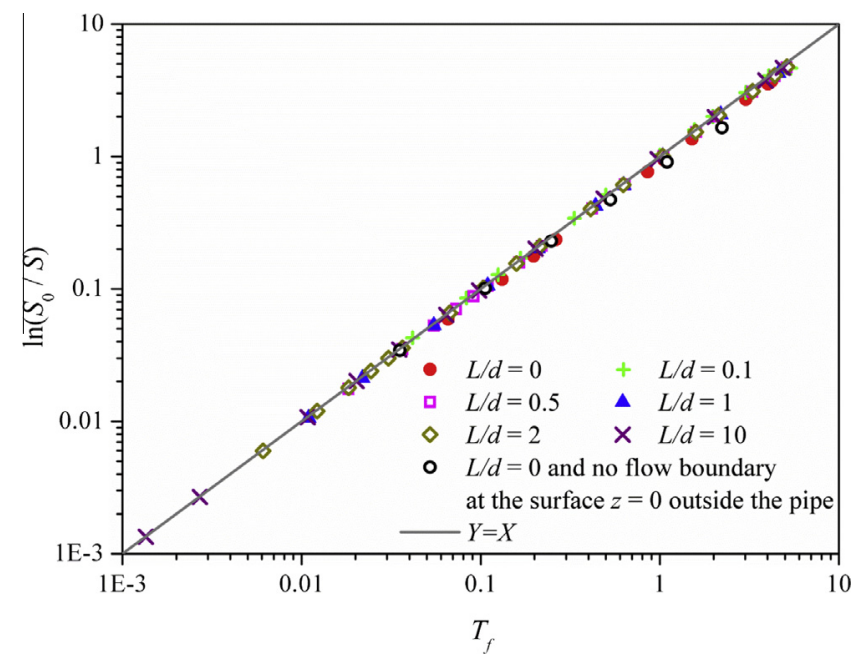

Fig. 3. Simulated $\ln \left(S_{0} / S\right)$ versus dimensionless time $\left(T_{f}\right)$ for an isotropic medium.

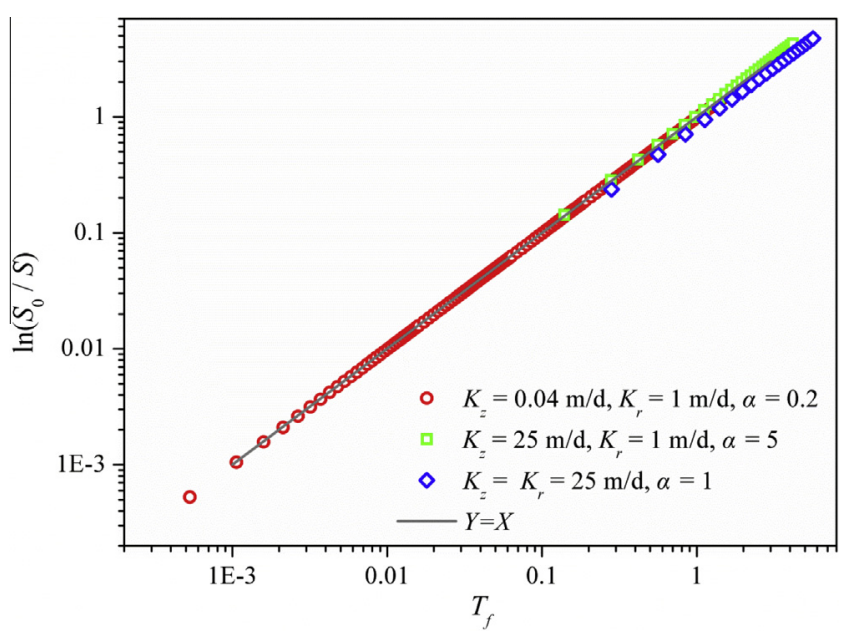

Fig. 4. Simulated $\ln \left(S_{0} / S\right)$ versus dimensionless time $\left(T_{f}\right)$ for an anisotropic medium and constant penetration depth $(L / d=5)$.

Fig. 5 shows the results of these simulations. As expected, every tested depth with internal resistance produces a value of estimated conductivity that is very close to the harmonic mean estimated at each given depth. The tests with zero internal resistance produce estimated values much closer to the intrinsic value of the conductivity at the tested depth. Thus, to study the vertical variability in hydraulic conductivity of bottom sediments, the testing accuracies can be improved by removing the sediments from the pipe. The tests with sediments inside the pipe produce estimated values of resistance that averaged up to the harmonic mean of the testing depth.

\section{Summary}

In this study, we developed a semi-analytical expression that accounts for flow in a bounded medium during falling head tests in a pipe penetrating the riverbed sediments. This expression considered the total hydraulic resistance of the flow through the sediments into and out of the pipe, thereby producing a generalized solution to the well-known Hvorslev (1951) formula (Eq. (25)). The developed expression was tested using the simulation results of a finite-element numerical model. Numerical comparisons with the proposed analytical solution exhibit good agreement between simulated and estimated hydraulic conductivity values for both isotropic and anisotropic media. Thus, the linear fitting of the ln $\left(S / S_{0}\right)$ curve versus test time and calculation of the $a(L)$ value from Eqs. (15) and (18) for known geometry produce a more accurate result than that of the Hvorslev formula when processing field test data. As illustrated in Fig. 2, the influence of the constant head boundary at the top of the sediments is visible for penetration depths that are less than two pipe diameters.

For sediments with ambient pre-test vertical head gradients, the non-steady state process of head recovery in the pipe due to natural differences between the hydraulic head in the surface water and the hydraulic head in the sediments at depth $L$ can affect the interpretation of the results. The magnitude of the errors in the results is generally unknown. To account for an existing vertical head gradient within the riverbed sediments would require special piezometers that measure very small head differences (e.g., Kennedy et al., 2007), capable of defining the ratio of $S_{0}$ to the natural head difference in the surface water and the sediments. Analytical analyses and numerical simulations show that for typical geometries associated with falling head test, i.e., $S_{0}$, penetration depth $L$ and pipe diameter $d$, and typical elastic storage of medium, 

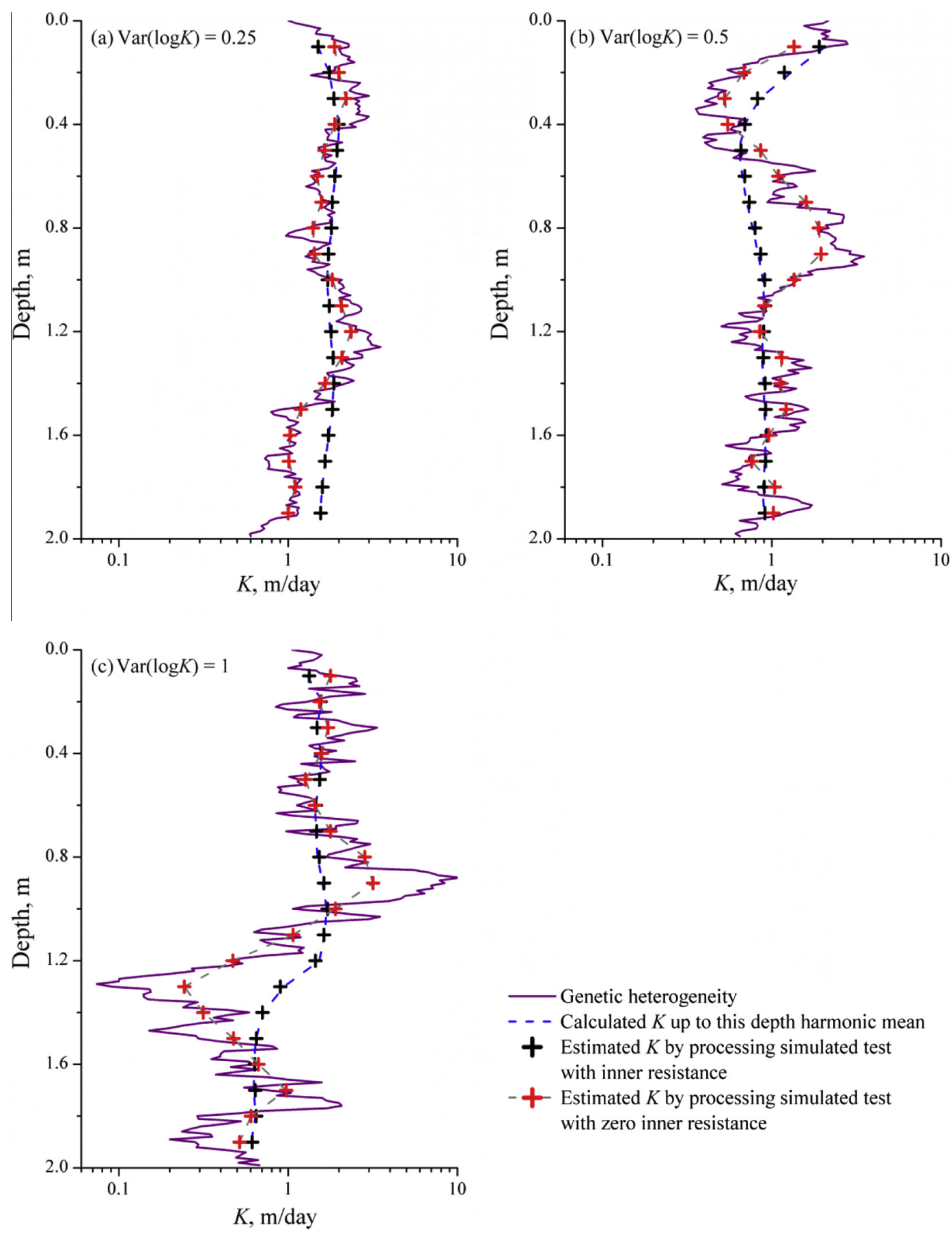

- Genetic heterogeneity

- - - Calculated $K$ up to this depth harmonic mean

+ Estimated $K$ by processing simulated test with inner resistance

- + Estimated $K$ by processing simulated test with zero inner resistance

Fig. 5. Results of processing the simulated tests in a heterogeneous layered medium.

the flow in riverbed sediments can be considered flow in incompressible medium, and the expression for hydraulic resistance does not change with the test time.

Finally, a set of simulations in layered, stochastically heterogeneous riverbed sediments was also performed and processed using the obtained expression to investigate the possibility of measuring vertical heterogeneity in sediments via a series of standpipe permeameter tests with different pipe penetration depths. The results show that tests with sediment inside the pipe yield estimated hydraulic conductivity that are close to the harmonic mean of the hydraulic conductivities of the riverbed sediments inside the pipe. However, to investigate the vertical variability in the hydraulic conductivity of the riverbed sediments, a more appropriate approach is to remove the sediment from the pipe. Thus, tests without sediments inside the pipe provide more information regarding vertical heterogeneity in the riverbed. Practically, after penetration of the pipe to a given depth $L$, this type of test requires the use of a drilling spoon or bailer with a diameter slightly smaller than that of the pipe to extract the sediments. The results of this study can be used to provide more accurate estimates of riverbed hydraulic conductivity and to provide guidance for the field design of falling head tests. 


\section{Acknowledgments}

This research was supported by grants from the National Natural Science Foundation of China (No. 41301025), and the NSFC-RFBR Programme 2015-2016 (Nos. 41511130025 and 1555-53010 ГФЕН_a). The authors gratefully acknowledge the Editor, Corrado Corradini, the Associate Editor, Philip Brunner, and the anonymous reviewers for their valuable comments and suggestions that have led to substantial improvements over an earlier version of the manuscript. Special thanks to Paul Davis, the head of the EnviroLogic Inc., for his helpful discussion and manuscript editing.

\section{References}

Bear, J., 1979. Hydraulics of Groundwater. McGraw-Hill Book Company, New York, $569 \mathrm{p}$.

Baxter, C., Hauer, F.R., Woessner, W.W., 2003. Measuring groundwater-stream water exchange: new techniques for installing minipiezometers and estimating hydraulic conductivity. Trans. Am. Fish. Soc. 132 (3), 493-502.

Burnette, M.C., Genereux, D.P., Birgand, F., 2016. In-situ falling-head test for hydraulic conductivity: evaluation in layered sediments of an analysis derived for homogenous sediments. J. Hydrol. 539, 319-329.

Calver, A., 2001. Riverbed permeabilities: information from pooled data. Ground Water 39 (4), 546-553.

Carslaw, H.S., Jaeger, J.C. (Eds.), 1959. Conduction of Heat in Solids. Clarendon Press, Oxford.

Chen, X., 2000. Measurement of streambed hydraulic conductivity and its anisotropy. Environ. Geol. 39 (12), 1317-1324.

Chen, X., Chen, X., 2003. Stream water infiltration, bank storage, and storage zone changes due to stream-stage fluctuations. J. Hydrol. 280 (1-4), 246-264.

Chen, X., Song, J., Wang, W., 2010. Spatial variability of specific yield and vertical hydraulic conductivity in a highly permeable alluvial aquifer. J. Hydrol. 388 (34), 379-388.

Cheong, J.-Y. et al., 2008. Estimating hydraulic conductivity using grain-size analyses, aquifer tests, and numerical modeling in a riverside alluvial system in South Korea. Hydrogeol. J. 16 (6), 1129-1143.

Constantz, J., 1998. Interaction between stream temperature, streamflow, and groundwater exchanges in alpine streams. Water Resour. Res. 34 (7), 16091615.

Dunkerley, D.L., 2008. Bank permeability in an Australian ephemeral dry-land stream: variation with stage resulting from mud deposition and sediment clogging. Earth Surf. Proc. Land. 33 (2), 226-243.

Fleckenstein, J.H., Krause, S., Hannah, D.M., Boano, F., 2010. Groundwater-surface water interactions: new methods and models to improve understanding of processes and dynamics. Adv. Water Resour. 33 (11), 1291-1295.
Genereux, D.P., Leahy, S., Mitasova, H., Kennedy, C.D., Corbett, D.R., 2008. Spatial and temporal variability of streambed hydraulic conductivity in West Bear Creek, North Carolina, USA. J. Hydrol. 358 (3-4), 332-353.

Hatch, C.E., Fisher, A.T., Ruehl, C.R., Stemler, G., 2010. Spatial and temporal variations in streambed hydraulic conductivity quantified with time-series thermal methods. J. Hydrol. 389 (3-4), 276-288.

Hiscock, K.M., Grischek, T., 2002. Attenuation of groundwater pollution by bank filtration. J. Hydrol. 266 (3-4), 139-144.

Hvorslev, M.J., 1951. Time lag and Soil Permeability in Groundwater Observations. Waterways Experiment Station Bulletin, vol. 36. US Army Corps of Engineers, Vicksburg, MS.

Kalbus, E., Reinstorf, F., Schirmer, M., 2006. Measuring methods for groundwatersurface water interactions: a review. Hydrol. Earth Syst. Sci. 10 (6), 873-887.

Kennedy, C.D., Genereux, D.P., Corbett, D.R., Mitasova, H., 2007. Design of a light-oil piezomanometer for measurement of hydraulic head differences and collection of groundwater samples. Water Resour. Res. 43 (9), W09501. http://dx.doi.org/ 10.1029/2007WR005904.

Klammler, H., Hatfield, K., Nemer, B., Mathias, S.A., 2011. A trigonometric interpolation approach to mixed-type boundary problems associated with permeameter shape factors. Water Resour. Res. 47 (3), W03510.

Landon, M.K., Rus, D.L., Harvey, F.E., 2001. Comparison of instream methods for measuring hydraulic conductivity in sandy streambeds. Ground Water 39 (6), 870-885.

Lee, B.-J., Lee, J.-H., Yoon, H., Lee, E., 2015. Hydraulic Experiments for Determination of In-situ Hydraulic Conductivity of Submerged Sediments. Sci. Rep., 5.

Lekhov, M.V., 2015. Modeling of wells in cross section of an unconfined aquifer. The 1WELL program. Eng. Geol. (3), 52-64 (in Russian with English abstract)

Min, L., Yu, J., Liu, C., Zhu, J., Wang, P., 2013. The spatial variability of streambed vertical hydraulic conductivity in an intermittent river, northwestern China. Environ. Earth Sci. 69 (3), 873-883.

Neuman, S.P., 1975. Analysis of pumping test data from anisotropic unconfined aquifers considering delayed gravity response. Water Resour. Res. 11 (2), 329342.

Shanafield, M., Cook, P.G., 2014. Transmission losses, infiltration and groundwater recharge through ephemeral and intermittent streambeds: a review of applied methods. J. Hydrol. 511, 518-529.

Silvestri, V., Abou-Samra, G., Bravo-Jonard, C., 2012. Shape factors of cylindrical piezometers in uniform soil. Ground Water 50 (2), 279-284.

Taylor, A.R., Lamontagne, S., Crosbie, R.S., 2013. Measurements of riverbed hydraulic conductivity in a semi-arid lowland river system (Murray-Darling Basin, Australia). Soil Res. 51 (5), 363-371.

Wang, P., Pozdniakov, S.P., Shestakov, V.M., 2015. Optimum experimental design of a monitoring network for parameter identification at riverbank well fields. J. Hydrol. 523, 531-541.

Warrick, A.W., Broadbridge, P., Lomen, D.O., 1992. Approximations for diffusion from a disk source. Appl. Math. Model. 16 (3), 155-161.

Winter, T.C., Harvey, J.W., Franke, O.L., Alley, W.M., 1998. Ground Water and Surface Water: A Single Resource. 